\title{
25 The exchange of support and financial assistance: differences in exchange patterns and their implications for ageing well
}

- Network members play different roles in the exchange of different kinds of help

- Regional differences prevail in the exchange of support with social network members

- Financial transfers with network members are correlated with greater well-being

\subsection{The exchange of support and financial assistance among older European adults}

Human beings live and function within complicated exchange networks from which they derive a range of resources and supports that are necessary for them to thrive. Within their exchange networks, people tend to trade time and money as well as information, values and space (Litwin et al. 2008). Such exchange has particular importance in the latter half of life, insofar as both the needs that people have and their resources change as they age. Moreover, the exchange of support is a crucial component of intergenerational solidarity. It also constitutes a key source of motivation for older people to remain active and to live independently in the community (Gray 2009, Chiatti et al. 2013).

Much of the literature places the exchange network within the larger domain of the social network, that is, the collection of social ties that people generally maintain and from which they receive various kinds of assistance. However, some recent studies distinguish between exchange networks and personal social networks. The latter are comprised by the most meaningful relationships that people maintain, the social ties that they consider to be their confidants. This distinction between personal social networks and exchange networks is important because these two social entities may have different implications for well-being in late life. That is, personal social networks and exchange networks might work in different ways and they may yield different outcomes (Cheng et al. 2011).

This chapter focuses on the exchange of practical support and financial assistance among older European adults and the role of the personal social network in such exchanges. These particular modes of exchange, time and 
money, are the two dominant kinds of transfers. Financial transfers comprise the exchange of money and goods; time transfers include provision of services such as physical assistance with daily activities (Litwin 2004). The research reported here examines the role of the personal social network in the exchange of this support. We seek to clarify the extent to which different types of exchange are carried out with social network members as opposed to the exchange that takes place with other persons, those not considered to be confidants. We also seek to learn whether exchange with members of the personal social network is differentially associated with selected well-being outcomes than exchange with others.

Although exchange is a universal phenomenon, it may well be shaped by differing cultural norms, values and customs. Patterns of exchange may also be tempered by social policy and by the varying availability of public services, such as those provided through the welfare state. This latter point is particularly relevant for intergenerational exchange, insofar as the exchange of informal support across generations may be encouraged or, conversely, "crowded out" by existing formal services and benefits (Kohli et al. 2009). We consider the question of cultural context in terms of country differences. That is, do exchange patterns differ by country?

Personal social network is measured in the SHARE Wave 4 questionnaire by a unique module that identifies the people with whom respondents discussed matters of importance to them during the previous twelve months. This form of network mapping, by means of a name generator, allows the designation of respondents' closest ties, that is, their unique collection of confidants (Litwin et al. 2013). Given that social contacts with significant others, in this case - the members of the personal social network, are generally more meaningful than contacts with others, it may also be assumed that exchange with members of the personal social network are more meaningful vis a vis well-being outcomes. We hypothesise, therefore, that exchanges with the personal social network will yield more positive associations with selected well-being outcomes than exchanges with others.

As for the cultural context, it may be assumed that exchanges with intimate others, like members of the personal social network, are more significant in settings where fewer formal support alternatives exist. Broadly speaking, we might expect such exchanges to be more meaningful in the Southern and Eastern countries of Europe, where welfare state services are less available, than in the North and the West of the continent. We hypothesise, therefore, that exchange with members of the personal social network will have stronger associations with selected well-being outcomes among the respondents from the Southern and Eastern countries. 


\subsection{How we measured the modes of exchange}

The overall study sample for the inquiry reported here included 56,755 respondents aged 50 and older. However, insofar as the SHARE questionnaire routes the questions differentially, not all respondents were asked all of the questions. The probes on personal care within the household were addressed only to respondents who resided in households larger than one, by definition $(\mathrm{N}=44,485)$. Questions about assistance given or received to or from someone elsewhere were routed to the family respondent, i.e., one designated respondent per household $(\mathrm{N}=39,335)$. Queries on financial transfers were answered, in turn, by the selected financial respondent, again, one person per household $(\mathrm{N}=38,888)$.

The analysis addressed three key areas of exchange: 1) personal care that is given to or received from someone within the household, 2) assistance of any kind that is given to or received from someone elsewhere (outside the household), and 3) the provision or receipt of financial transfers. These variables were operationalised as follows. Personal care from/to a member of the household refers to regular help (almost daily) of at least three months in tasks such as washing, dressing and getting out of bed. Assistance from/to someone elsewhere reflects personal care, practical help in the home, or help with paperwork or legal matters. It should be noted, therefore, that the first variable measures only personal care given by or to a co-resident in the household whereas the second variable addresses any kind of help provided by or to anyone who does not co-reside in the same household with the recipient. The third exchange variable - financial transfer - reflects either of two monetary exchanges: 1) assistance of at least 250 Euros, or 2) a large gift (5,000 Euros or more). All three of the exchange variables were dichotomised to reflect receipt (or not) and provision (or not) of each form of support.

As noted earlier, identification of the personal social network was obtained through the use of a name generator. The naming of confidants allowed subsequent queries as to the nature of the relationship with the person to whom help was given and from whom help was received. Specifically, this mechanism allowed the creation of the variables necessary for the current inquiry: help given and received in each of the three areas of support, to/from a network member or to/from a person not considered to be part of the personal social network. The linkage that made this possible was carried out only for the cases in which support was actually exchanged (given or received).

Two well-being outcome variables were considered. The CASP scale reflects functional well-being on twelve different items and is measured on a scale of 12-48 (Hyde et al. 2003). Life satisfaction was measured on a single global probe yielding a score of 1-10. The higher the scores in both cases, the greater was the respondent's state of well-being. 
Control variables taken into account included selected demographic background and health variables. The background variables included age, gender, number of children, marital status, education level and perceived income adequacy. The health variables were the number of chronic conditions, the number of physical symptoms, limitations in activities of daily living (ADL) and limitations in instrumental activities of daily living (IADL).

\subsection{How we considered support types and directions}

The first analysis considered the support types and directions that were more dominant among the social network members and those which were observed more frequently among the more distant relationships. In the second phase of the inquiry, logistic regressions identified country differences in relation to these same types of support. We should note that country differences have already been found in the provision and receipt of transfers (Attias-Donfut et al. 2005). This phase of the current analysis considered whether there were any country differences vis a vis the role of the personal social network in the respective transfers, after controlling for demographic and health variables. The third part of the study employed linear regressions to assess the relationships between each support exchange type and the well-being of the respondents, controlling for demographic and health variables and for country differences. The descriptive analysis employed normalised weights. The regressions did not.

\subsection{Exchange of support with confidants}

On the whole, about one third of the households gave money to others (31\%) and a quarter gave personal or practical support to someone not in the household. Somewhat less prevalent were the receipt of personal or practical support from someone not in the household (20\%) and the receipt of money (17\%). The least common types of exchange were the receipt and provision of personal care from/ to someone in the same household ( $12 \%$ and $8 \%$, respectively).

Investigation into the proportion of social network members involved in these transfers presents a somewhat more complex picture (Figure 25.1). The vast majority of respondents who received money did not receive it from a person they had named in their personal social network (80\%). In contrast, almost one half of those who gave financial support did so to someone they considered to be a member of their confidant network. Additional analysis (not shown in the figure) revealed that this gap exists in most countries. 
A similar picture emerges in relation to support exchange with someone not in the household (elsewhere). Only about a third gave this kind of help to a personal social network member, on average, ranging from 17 per cent in Spain to 44 per cent in Hungary. In comparison, more than half of those who received help from elsewhere received such help from someone in their personal social network, on average. We note, nevertheless, that the diversity between countries in relation to the receipt of help from elsewhere was high, ranging from 35 per cent in the Netherlands to 78 per cent in Italy.

As for personal care given in the household, slightly more than half of those giving such help (a small minority overall) gave the help to a network member. This ranged from 39 per cent in Portugal to 74 per cent in Hungary. In contrast, the proportion of those receiving personal care in the household who received such care from someone considered to be a network member was much greater (about $80 \%$, on average). Ranging from 72 per cent in Belgium to 93 per cent in Hungary, this high degree of network-provided support may be due to the fact that most respondents cited a spouse in their personal social network and the spouse is generally the main provider of personal care in the household.

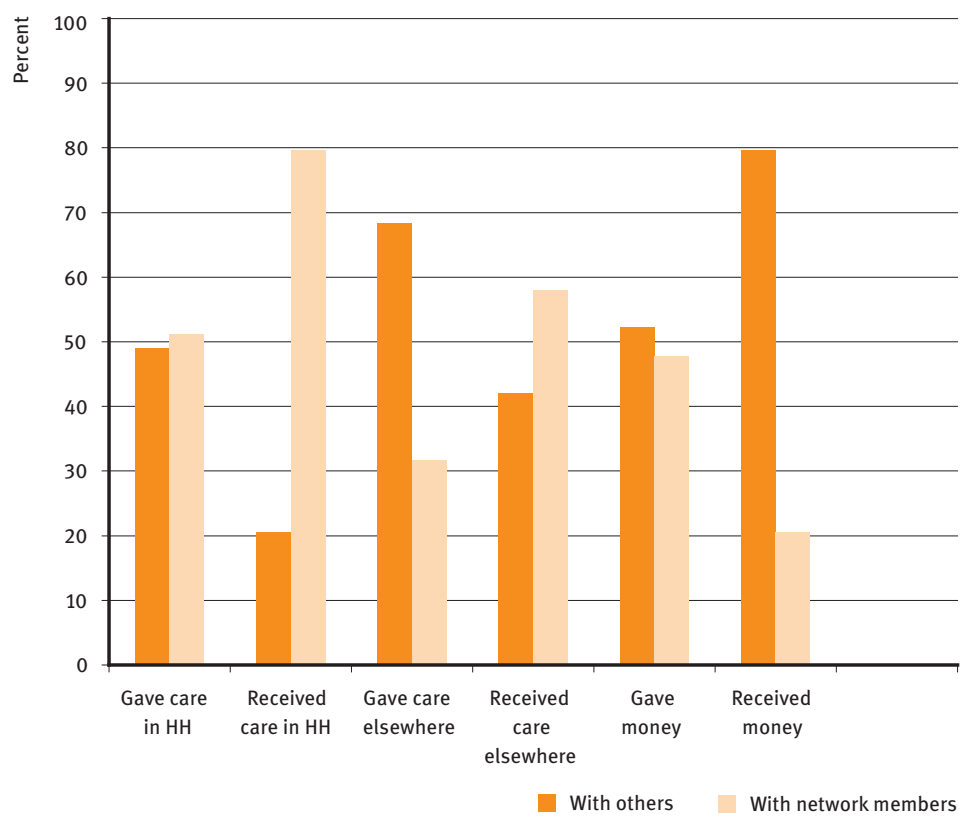

Figure 25.1: Exchange of support with network members and with others (weighted) Notes: Unweighted observations: Gave care in $\mathrm{HH}(n=3,580)$, Received care in $\mathrm{HH}(n=2,419)$, Gave care elsewhere $(n=10,023)$, Received care elsewhere $(n=7,674)$, Gave money $(n=11,888)$, Received money $(n=8,169)$

Source: SHARE Wave 4 release 1 


\subsection{Country differences in the exchange of support with social network members}

In order to more clearly substantiate the country differences in relation to the probability of exchange with members of the personal social network, each of the dichotomous exchange outcomes (transfers from or to network member as opposed to transfers from or to other relationships) was regressed on country, controlling for demographic background and health. Effect coding was employed to ascertain the country effects. The results of the respective analyses are presented in Figures 25.2-25.4. Each figure presents the odds ratios of provision and receipt of the respective types of support exchange with a personal social network member, as opposed to exchange with a non-network member. The countries that showed significant differences in this respect are marked by asterisks.

Figure 25.2 presents the odds ratios, by country, for the provision and receipt of personal care in the household to and from someone in the personal network. It may be seen from the figure that among those who gave help in the household, the respondents from France, Spain and Italy had a lower probability of giving such help to a social network member than the general country mean. In contrast, the respondents from the Netherlands, Austria and Hungary each had a higher probability of giving such help to a social network member. Country variations were also found in relation to the receipt of such in-house support from a confidant. The Belgians and the French were less likely to have received personal care from someone in their personal social network. The Austrians and the Hungarians had a higher probability of having received such help from a network member.

The probability of exchange of personal and practical help with a social network member elsewhere is summarised in Figure 25.3. As the figure reveals, respondents from the Northern and Western countries, for the most part, were less likely to have received help from members of their personal social networks outside their households, than the level reflected in the general country mean. Respondents from the South and the East, in comparison, were generally more likely to have received such help. Those from the Czech Republic and Hungary were more likely to have given such help, while those from Spain and Portugal were less likely. Austrian respondents were unique among the Western countries in that they were likely to have both given and received help to and from members of the personal social network outside their households. The Italians were more likely to have received such help, but not to have given it. 


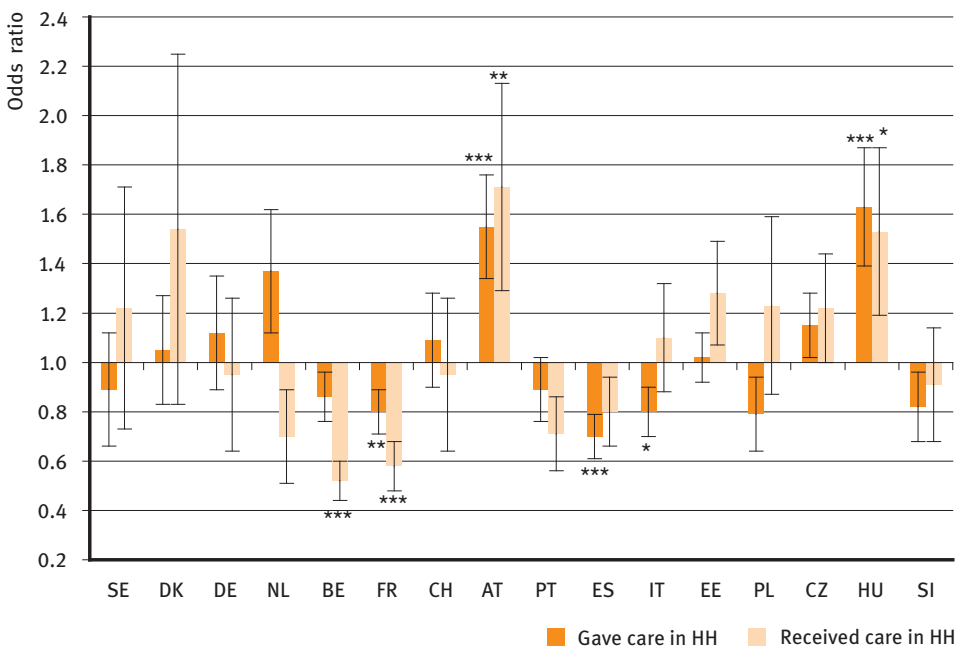

Figure 25.2: Country differences in the exchange of support with network members in the $\mathrm{HH}$ : Odds ratios and standard errors

Significance: ${ }^{*}=10 \%$; ${ }^{\star \star}=5 \%$; ${ }^{\star \star \star}=1 \%$

Notes: Gave care in $\mathrm{HH}(\mathrm{n}=3,580)$, Received care in $\mathrm{HH}(\mathrm{n}=2,419)$

Source: SHARE Wave 4 release 1

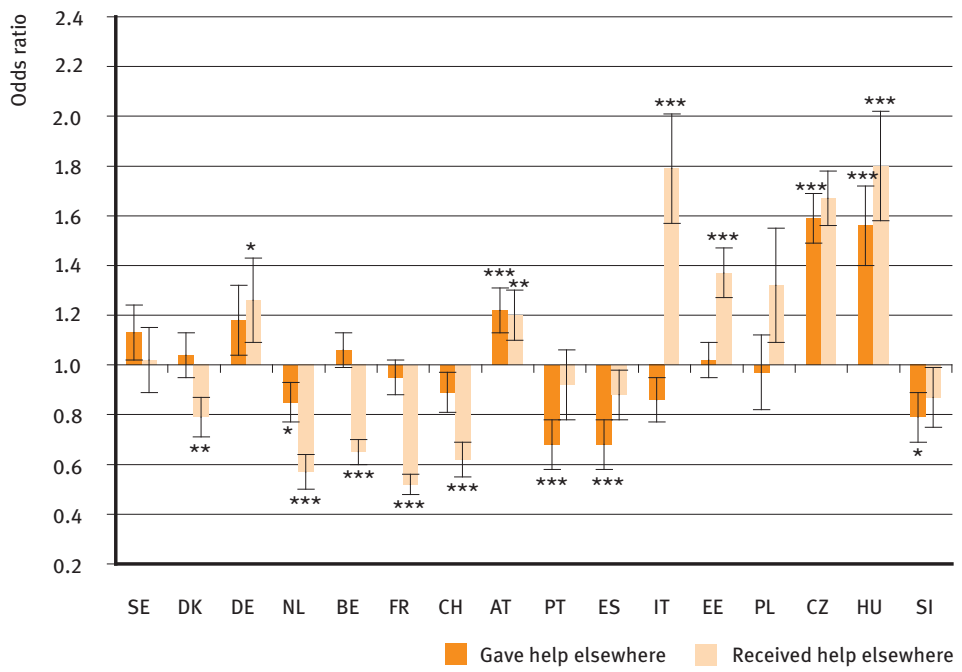

Fig. 25.3: Country differences in the exchange of support with network members elsewhere:

Odds ratios and standard errors

Significance: * $=10 \% ;{ }^{* *}=5 \% ;{ }^{* *}=1 \%$

Notes: Gave care elsewhere $(n=10,023)$, Received care elsewhere $(n=7,674)$

Source: SHARE Wave 4 release 1 
Figure 25.4 presents the findings in relation to the exchange of financial support. As is evident in the figure, respondents from the North and West were, for the most part, less likely to have received a financial transfer from someone they considered to be a member of their personal social network. In contrast, the respondents from the East, again for the most part, were more likely to have received a financial transfer from a social network member. The patterns for giving financial support were more varied. A majority of the countries were less likely to give money to a social network member (Sweden, Denmark, the Netherlands, Belgium, France, Switzerland, Spain and Slovenia). However, respondents from Austria, Portugal, Estonia, Poland and Hungary had higher expected odds of giving such financial support.

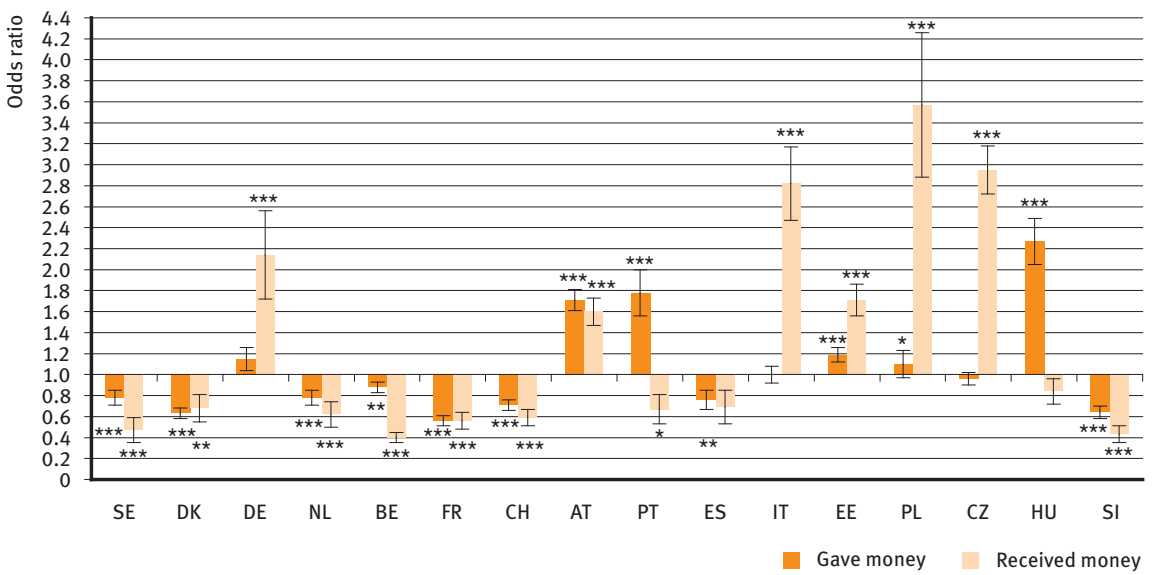

Figure 25.4: Country differences in the exchange of financial support with network members: Odds ratios and standard errors

Significance: ${ }^{*}=10 \% ;{ }^{* \star}=5 \% ;{ }^{* \star *}=1 \%$

Notes: Gave money $(n=11,888)$, Received money $(n=8,169)$

Source: SHARE Wave 4 release 1

\subsection{Patterns of exchange of support with social network members}

Looking at the composite picture, that is, the tendency of respondents in different countries to engage in exchange of all kinds with people they consider to be their confidants, shows a number of patterns. Based upon the findings presented in the figures, we grouped the countries in which the respondents were more likely to have engaged in substantial exchange with members of their personal social network. 
We considered a greater likelihood of exchange to have occurred when countries revealed significantly higher or lower odds ratios in two or more of the three realms of exchange: personal care within the household, help from/to someone elsewhere and/or financial assistance. This was done both for support provision and receipt.

The first pattern of note is that respondents from France and the Netherlands were less likely to have engaged in exchange with their most intimate social network, that is, with their named confidants. This was true for both giving and receiving. It seems, therefore, that the older cohorts in these particular countries tend not to exchange practical care and assistance of any kind with those they deem to be their close personal ties.

Another pattern worthy of attention is that respondents in two countries - Spain and Slovenia - were less likely to have given substantial support to members of their closest personal social network. Yet, the respondents from these same countries were not less likely to have received substantial support from their confidants. A more definitive pattern was observed among the respondents from Austria and Hungary. They were more likely to have received substantial assistance from their closest ties and to have provided help to close ties in most types of help (Hungary), or in all of them (Austria). This particular set of findings suggests that the older cohorts in Austria and Hungary, unlike their counterparts in France and the Netherlands - actively engage in exchange with the members of the personal social networks.

\subsection{The exchange of support with members of the personal social network and well-being}

In order to clarify whether there are indeed differential implications of the exchange of support with members of one's personal social network for one's well-being in the second half of life, in comparison to the exchange of help with others, we examined the association of such exchange with two outcome measures - the CASP Scale and a life satisfaction measure. For this purpose, each of the well-being outcomes was regressed on the six types and directions of transfer, controlling for the demographic background and health variables mentioned earlier, and for country. We employed effect coding to observe the differences among the country mean scores on the respective well-being outcomes, in relation to the overall unweighted sample mean. As recalled, each dichotomous exchange variable in the analysis reflected the exchange of the help in question with a social network member as opposed to the exchange of help with others. 
As can be seen in Figure 25.5, only the financial transaction type was accompanied by better well-being when the persons engaged in the exchange were members of the personal social network. Respondents who gave money to their social network members had higher CASP scores and higher life satisfaction than respondents who gave money to more distant relationships, persons who were not considered to be confidants. In addition, the receipt of money from a social network member rather than from someone else was also correlated with higher life satisfaction. These findings are of particular note when considering the distribution of the financial transfers with confidants versus transfers with more emotionally distant relationships. The data show that while the vast majority who had received financial assistance got such help from persons who were not considered to be members of their personal social network, those who did engage in financial exchange with their most intimate relationships experienced greater life satisfaction, controlling for all other variables. As for the other types of transactions, Figure 25.5 shows that there was no difference in the well-being level between those who exchanged practical support and personal care with persons considered to be members of their social network and with others.

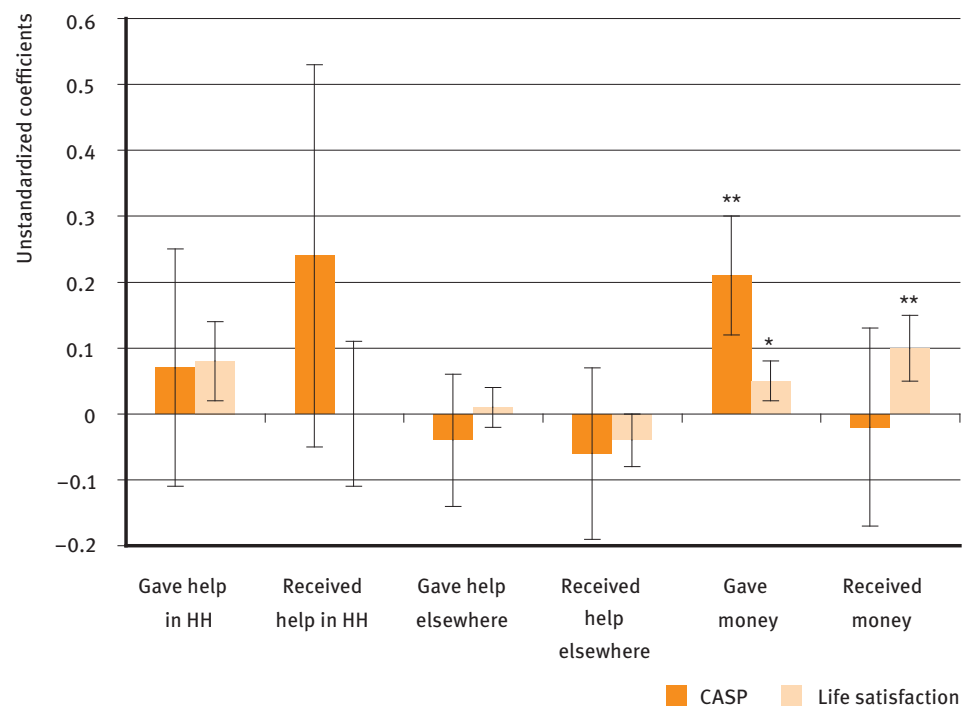

Figure 25.5: The association between exchange of support with network members versus the exchange of support with others and well-being: Unstandardised coefficients and standard errors Significance: ${ }^{*}=10 \% ;{ }^{* \star}=5 \% ;{ }^{\star \star \star}=1 \%$ Notes: Gave care in HH $(n=3,580)$, Received care in $\mathrm{HH}(n=2,419)$, Gave care elsewhere $(n=10,023)$, Received care elsewhere $(n=7,674)$, Gave money $(n=11,888)$, Received money $(n=8,169)$ Source: SHARE Wave 4 release 1 


\subsection{The role of the social network in the exchange of help and its correlation with well-being}

This study examined the role of the personal social network in the exchange of practical care, help and financial assistance, and its correlation with well-being. The results revealed that the exchange of support with someone within the household and the receipt of practical or personal care from someone elsewhere indeed took place mainly with members of the personal social network. In comparison, the receipt and giving of money and providing support to someone not in the household occurred more frequently with those who were not considered to be part of the personal social network.

The findings further revealed that there were relatively few country differences in the exchange of support with someone within the household. There were also few country differences in the giving of help to a confidant elsewhere. But sundry differences were observed in the other areas of exchange, suggesting that this area of inquiry requires further exploration.

Our first study hypothesis, that exchange with personal social network yields more positive associations with well-being than exchanges with others, was only partly supported. This was observed in relation to the financial transfers, but not for the exchange of practical help and personal care. It seems from the data, therefore, that people are more sensitive to financial exchanges than to other kinds of assistance given or received.

The second hypothesis also received partial confirmation, this time vis a vis help and care exchanged outside of the household. The findings showed, generally, that respondents from Northern and Western countries were less likely to have received such help while those from Southern and Eastern countries were more likely. These regional differences parallel the public service divide. In the North and the West, where public services are more generous, the exchange of informal assistance with the personal social network was less likely. In the South and the East, where public services are less available, the exchange of informal assistance with the personal social network was more likely.

Despite these two key findings, more still needs to be learned about the associations that exist between personal social networks and exchange in late life. The unique cases identified in this analysis, such those observed in Austria, Hungary, and to a lesser degree, Italy, indicate that more research in this domain and new explanatory paradigms are required. The innovative panel data that SHARE makes available to social scientists will undoubtedly aid in furthering this goal. 


\section{References}

Attias-Donfut, Claudine, Ogg, Jim, Wolff, Francois-Charles (2005): “European patterns of intergenerational financial and time transfers”. In: European Journal of Aging 2, p. 161-173.

Cheng, Sheung-Tak, Li, Kin-Kit, Leung, Edward, Chan, Alfred (2011): "Social exchanges and subjective well-being: do sources of positive and negative exchanges matter?" In: Journals of Gerontology Series B - Psychological Sciences and Social Sciences 66, p. 708-718.

Chiatti, Carlos, Melchiorre, Maria Gabriella, Di Rosa, Mirko, Principi, Andrea, Santini, Sara, Dohner, Hanneli, Lamura, Giovanni (2013): “Family networks and supports in older age”. In: Phellas, Constantinos (Ed.): Aging in European societies. New York: Springer Science+Business Media, p. 133-150.

Gray, Anne (2009): “The social capital of older people”. In: Ageing \& Society 29, p. 5-31.

Hyde, Martin, Wiggins, Richard, Higgs, Paul, Blane, David (2003): “A measure of quality of life in early old age: the theory, development and properties of a needs satisfaction model (CASP-19)”. In: Aging \& Mental Health 7, p. 186-194.

Kohli, Martin, Hank, Karsten, Künemund, Harald (2009): “The social connectedness of older Europeans: patterns, dynamics and contexts”. In: Journal of European Social Policy 19, p. 327-340.

Litwin, Howard (2004): "Intergenerational exchange and mental health in later-life - the case of older Jewish Israelis". In: Aging \& Mental Health 8, p. 196-200.

Litwin, Howard, Stoeckel, Kimberly, Roll, Anat, Shiovitz-Ezra, Sharon, Kotte, Markus (2013): “Social network measurement in SHARE Wave 4". In: Frederic Malter \& Axel Börsch-Supan (Eds.): SHARE Wave 4: innovations \& methodology. Munich: MEA. Max-Planck-Institute for Social Law and Social Policy.

Litwin, Howard, Vogel, Claudia, Kunemund, Harald, Kohli, Martin (2008): “The balance of intergenerational exchange: correlates of net transfers in Germany and Israel”. In: European Journal of Ageing 5, p. 91-102. 